\title{
Validating algorithms for calculation of the shape of a flexible blade
}

\author{
A. de Smit ${ }^{1}$, A.J. Medland ${ }^{2}$, J.J. Broek ${ }^{1}$, A. Kooijman ${ }^{1}$, J.S.M. Vergeest ${ }^{1}$, I. Horváth ${ }^{1}$ \\ Corresponding author: A. de Smit \\ ${ }^{1}$ Faculty of Industrial Design Engineering, Landbergstraat 15, NL-2628CE Delft, \\ tel.:+31(0)152783788 \\ Fax.: $+31(0) 152781839$ \\ a.de.smit@io.tudelft.nl \\ ${ }^{2}$ Department of Mechanical Engineering \\ University of Bath \\ United Kingdom
}

\begin{abstract}
Methods and tools are developed to measure, analyze and compare physical and virtual blade shapes. An application is presented using measured data and the results of two blade shape calculation algorithms. A measuring method has been developed and a set-up for measuring physical blades was built. Tools were implemented to compare and evaluate the calculated and measured shapes. From the analysis of the measured data and the calculated results of the involved algorithms it could be conclude that none of the algorithms used initially could be applied directly for our shape calculations. The developed tools and methods have been found useful for the adaptation and fine tuning of the algorithms, which is demonstrated in two examples.
\end{abstract}

Keywords: Flexible blade; Shape measurement; Minimal strain energy curve; Shape Analysis, Thick layers; Polystyrene cutting.

\section{Introduction}

Shortening of production time of parts is an urgent necessity for automated prototyping during conceptual design of products. To make this possible a different approach than the currently available Rapid Prototyping (RP) technologies can be considered, namely an approach based on the principle of analogue manufacturing. This may offer a better operational effectiveness than the conventional Layered Manufacturing Technologies (LMT's), which are discrete technologies. In conventional LMT a physical 
model of a product is manufactured from a large number of small particles or thin layers, which are produced and placed sequentially. High-speed milling can also be described as a sequential particle handling technology, because small chips are sequentially milled off a stock, thus leaving the desired object. Due to this sequential handling of particles, the discrete technologies have unfavorable productivity features. An analogue tool typically is able to create a complex shape in one production step because the tool has a dedicated shape analogue to the desired product shape. The best example of an analogue production tool is a casting mould. Apart from preparation steps before- and finishing steps after the casting, this type of tool is capable to produce a shape in one action, namely the filling of the mould. However the range of products that can be produced by the tool is limited to one because the mould normally cannot be changed in shape during the production process. The ideal would be to develop a tool that can form itself according to the desired product shape so that this shape can be produced in one step. The authors are implementing an earlier proposed flexible cutting tool that can be actively shaped analogous to the geometry that must be produced, during the cutting process. This tool can be applied for cutting thick layers with freeform front surfaces and stacking a relatively small number of these layers can produce large free-formed shapes, as is illustrated in figure 1.

This principle of cutting free-form layers and stacking them to a prototype is called Free-Form Thick Layered Object Manufacturing (FFTLOM). By connecting the layers in a semi permanent manner it remains possible to edit the produced shapes by replacing one or more of these layers. This edit ability combined with an expected high production speed [1] makes the technology especially suitable for supporting conceptual design of large shapes. An expected drawback of the FF-

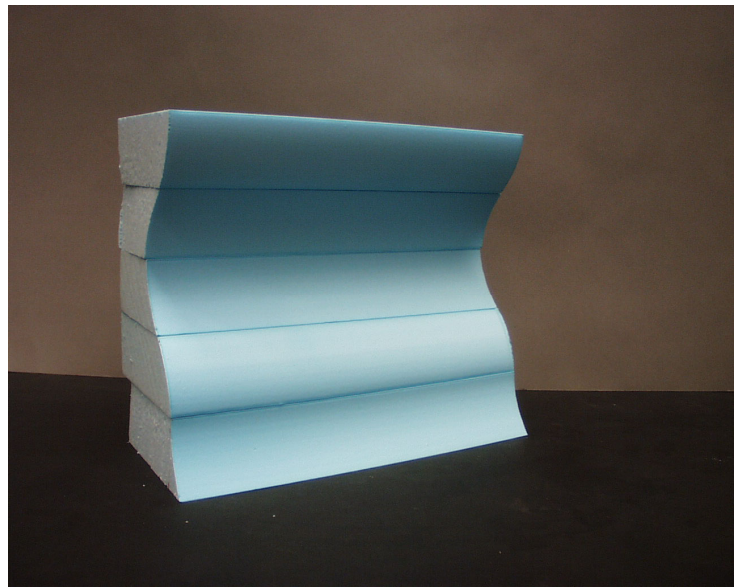

Figure 1. Example of a multi-layered stacked assembly.

TLOM technology is the limitation in possible detailing. In conventional LMT the layer thickness is the main constraint to the level of detail in the building direction. In FF-TLOM the minimal layer thickness will in general be much larger but the shape of the cutter can be adapted. However the form freedom of 
the flexible blade is physically constrained to smooth curves. Therefore the technology is more suited for smooth shapes than for highly detailed objects.

Thomas et al. [2], Hope et al. [3],de Jager [4] and Ahn [5] have published about the possibilities of Thick Layered Manufacturing and demonstrate systems that are able to produce layers with slanted front surfaces, thus achieving a first order approximation of the original freeform CAD model. In order to optimally reproduce the nominal CAD shape the principle of higher order approximation can be applied. Lee et al. [6] give a description on the implications of higher order approximation. In Hope et al. [3] the idea of a curved cutting tool is mentioned. Takeuchi et al. [7] describe the use of a cutting tool with a fixed conicoid shape, where different parts of the tool with different curvatures can be used sequentially. The ICA research group published a number of papers and articles in which the Freeform Thick Layered Object Manufacturing (FF-TLOM) was proposed. Horváth et al., [8], [9] and [10] describe the basic principles on which the technology is based.

\section{The problem to be solved}

The FF-TLOM technology uses a four degrees of freedom (4 DOF) flexible cutting tool. (Figure 2) The 4 DOF are used to control the shape of a flexible blade, which is the cutting part in this tool. The control parameters are the length $\mathbf{I}$ of the blade, the distance $\mathbf{d}$ between the two fixing points and two tangent settings $\boldsymbol{\alpha}$ and $\boldsymbol{\beta}$ at each end of the blade (Figure 3).

From these four parameters a shape is calculated that the blade will take assuming that it bends in a 2dimensional plane and can be represented by a twodimensional minimal strain energy curve. A calculation method must be selected that is able to calculate

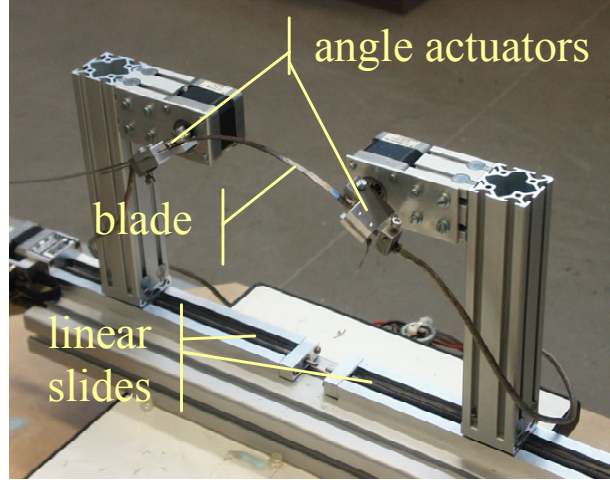

Figure 2. The tool that was build minimal strain energy curves based on these four parameters within an acceptable accuracy. The assumption that the blade behaves as a minimal strain energy curve must be verified by physical experiments, and if necessary the calculation method must be adapted to the measured physical reality. Furthermore the influence of control inaccuracies on the resulting blade accuracy must be determined. A sensitivity analysis must be performed on all the shaping parameters, thus defining the design constraints for a flexible cutting tool. The topics discussed in this paper are a) two shape calculation algorithms for 
2D minimal strain energy curves, b) measurement of physical shapes of bent flexible bars, c) analysis and evaluation of the calculated and measured results, d) interpretation of the found results in relation to physical tool implementation, e) adaptation of the algorithms in accordance with the physical reality.

\section{Algorithms used for the shape computation}

By definition the state of minimal elastic energy is reached for a curve if the length integral of the squared curvature of the curve is minimized. For calculation of a minimal energy curve various analytical [11], variational [6], and spline approximation algorithms [12] have been published. Most of the spline fitting algorithms minimize the square of the second derivative along the curve [13], [14]. An iterative approximating algorithm by Kallay was selected[15] and a newly developed application in an existing constraint-modeling environment [16]. For calculation of the blade shapes the following assumptions were made: a) the cutter blade can be modeled as a flexible bar, b) the cross section dimensions of the blade are much smaller than its length, c) no inelastic deformation takes place on the blade during shaping operations, d) the shape of the blade can be substituted by a planar curve, e) the blade is deformed by forces and moments acting only at its endpoints.

\subsection{The Kallay approximation method}

The first algorithm applied is developed for the calculation of space curves of minimal strain energy (MSE), and was first published in [15].

Instead of a continuous curve, a discrete approximation is used where the curve is represented by a polyline containing a limited number of points connected by straight linesegments of equal lengths. The algorithm calculates a polyline, which approximates the curve of minimal elastic energy. As the

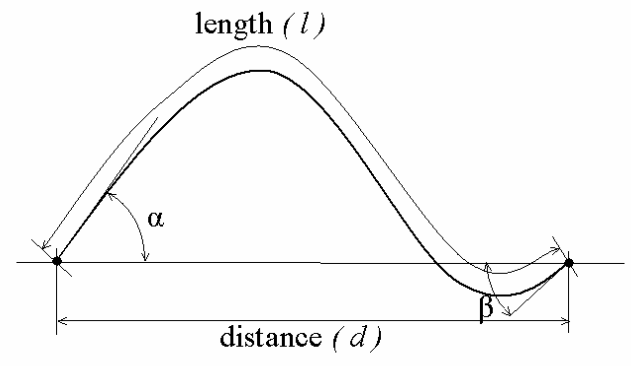

Figure 3. Shape control parameters algorithm searches a 3 dimensional solution space, this can result in a 3D curve, depending on the used constraints. In our case however a 3D curve is not applicable because our physical blade is kept planar due to its cross section shape. Kallay offers a solution in the form of a planar algorithm meant for cases where the 3D algorithm is trapped in a sub optimal planar situation. Figure 4 illustrates the basic principle 
starting from an initial saw tooth shaped polyline that is being deformed in an iterative manner until the state of minimal energy is reached under the given end conditions and blade length. The technique is described in Kallay [15] as a class of iterative geometric pivoting, based on the following principles. 1) A solution that meets the constraints is computed at each step. 2) The objective function improves with each step, and the iterations stop once it ceases to improve. 3) The solution at each step differs from that of the previous step only at a small number of joints between polyline segments. 4) The change at each step has a clear geometric significance (rotation, translation etc.).

Following Kallay, the first step in generating minimum strain energy (MSE) curve $e$ for given parameters $l, d, \alpha$ and $\beta$, is to set the first and last segment of the curve. If the points on $e$ bounding the segments are called $p_{i}$, where $0 \leq \mathrm{i} \leq m-1$ and if all segments are set to equal length $\mathrm{s}=l / m-1$, then we can set

$$
\begin{aligned}
& p_{0}=(0,0,0) \\
& \left.p_{1}=(s \cos (\alpha), s \sin (\alpha)), 0\right) \\
& p_{m-2}=(d-s \cos (\beta), \sin (\beta), 0) \\
& \left.p_{m-1}=(d, 0,0)\right) .
\end{aligned}
$$

The end segments will never change their position or orientation during the process. Note that, since the problem is purely planar, the $3 \mathrm{~d}$ coordinate is not relevant from here on. The remaining $m-3$ segments are distributed equally between the first and last segment, resulting in a saw tooth-like curve as shown in figure 4. After this initial step, the relative energy of the blade is decreased by repeatedly rotating one segment, or a number of contiguous segments of the blade, in the plane, about one of its endpoints, while keeping all segments of the polyline connected. The rotation is by some angle $\gamma$. For each new configuration of the segments the strain energy of the polyline is evaluated and the configuration with the lowest energy is retained. If for rotation angle $\gamma$ the energy does not decrease $\gamma$ is decreased by a certain relative amount. The procedure is repeated until $\gamma$ reaches a predefined lower bound.

The algorithm for generating the MSE curve $e$, given the parameters $d, l, \alpha, \beta$ and $m$ is as follows:

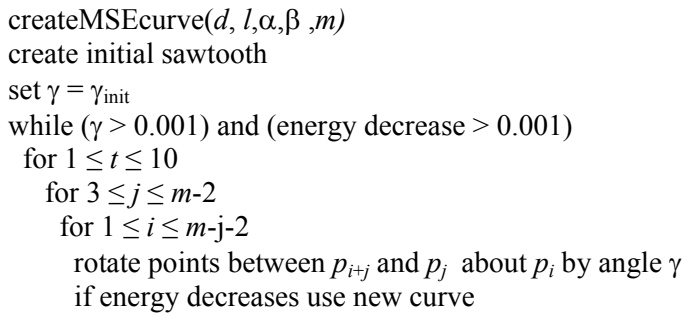




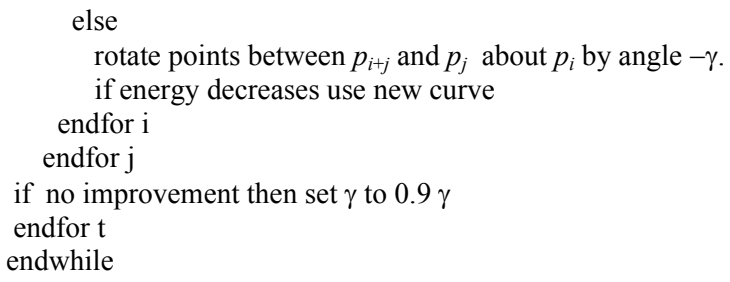

Figure 4 shows the initial sawtooth curve, a few steps in the generation process and the resulting MSE curve for $d=160, \alpha=30, \beta=45$.

A problem is that the used algorithm does not always result in an absolute minimum strain energy curve for the given parameters. The calculation can get trapped in a relative minimum, so that even the global shape of the blade is incorrect, or when the global shape of the blade is correct there can still be small differences in the calculation results, depending on the stop criteria that are used. Apparently the absolute minimal strain energy curve can only be approximated and almost never be exactly determined. For our experiments it was made sure that no relative minimum curves were used by a) using only shapes with maximally one inflection point and, b) visually checking the calculated shapes.

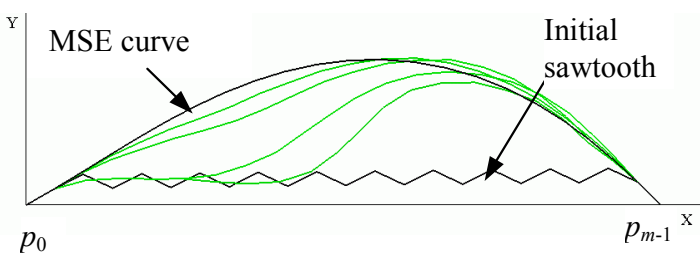

Figure 4. A few steps in the MSE curve generation

\subsection{Constraint based optimization method}

The second method that was applied for calculation of the shapes of the flexible blade is based on a constraint resolution strategy. For the actual calculations the constraint modeling software 'SWORDS' was used, which was developed by the Design Group at the University of Bath [16],[17].

In this case the problem is solved by a generic optimization process in which constraint rules (testable propositions) are used and direct search techniques are applied upon declared design variables. The constraint rules are assembled into resolving functions, which are themselves tested for their truths, calculated as the square root of the sum of the squares of the individual rules (with a true state being defined as zero).

In searching for a solution to the blade problem a geometric model is constructed from 20 line elements. These elements are created within their own model spaces (designated as $m m[1]$ to $m m[20]$ ) that are 
embedded and pivoted upon each other to form a chain (using a set of internal functions of the constraint modeling environment). This chain is grounded and pivoted at one end to a point declared within the world space. A second ground point is created upon which the resolution procedure should anchor the other end when a solution is found. Each of these ground points also carries a vector (of the same length as the chain sector lines) and set to the given start and end tangents.

In order to create the blade shape of minimum strain energy a function containing the element variables and the binding rules is created:

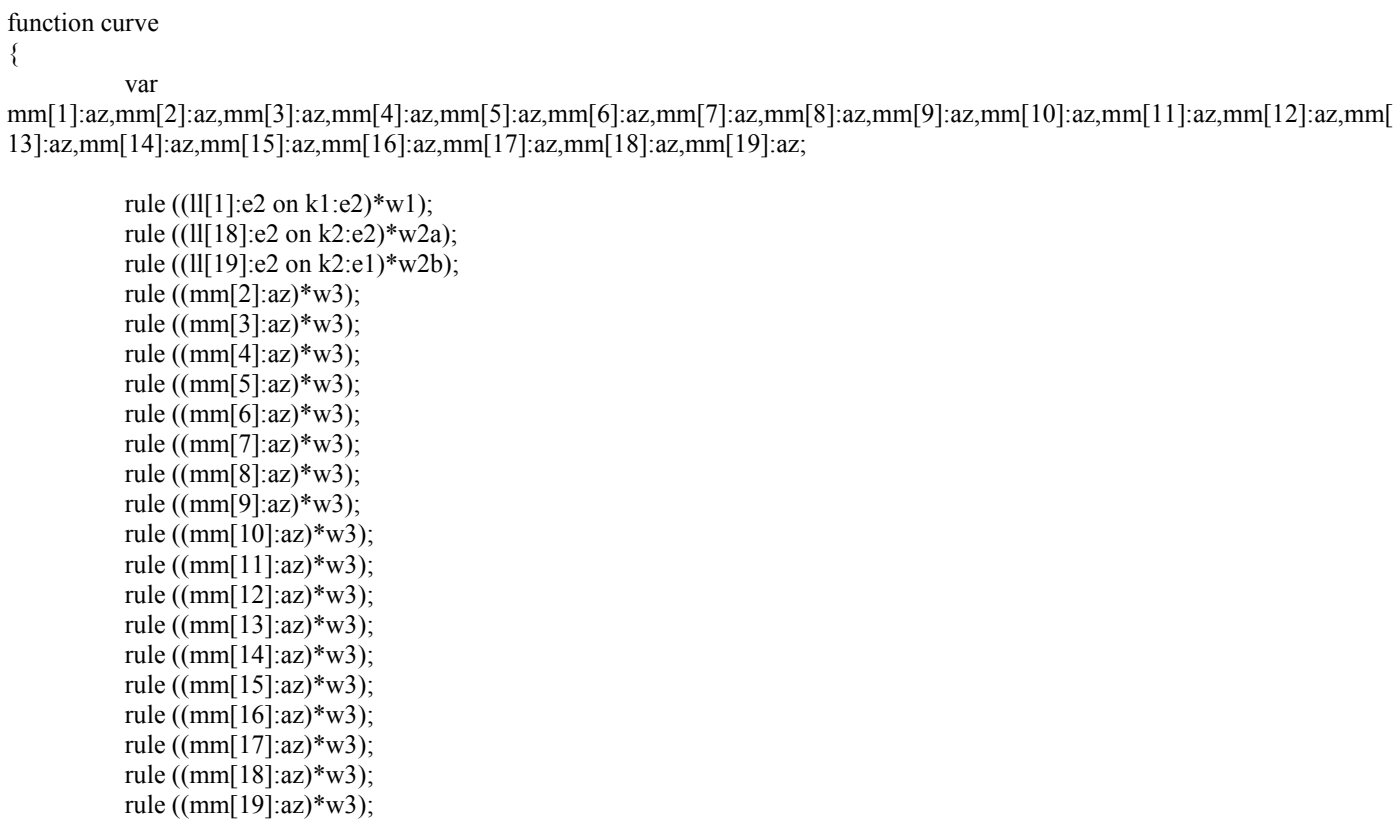

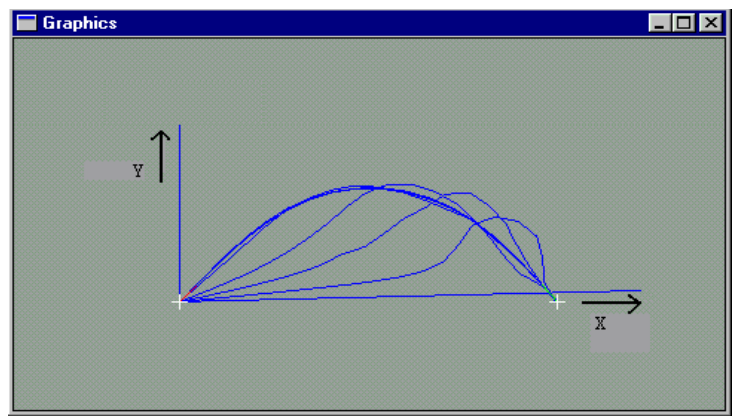

Figure 5. Iteration steps during constraint based optimization
The rules of this function can be considered in two groups. Three rules ensure that both the ends of the chain are on the ground points and the ends of the first and last segments are on the ends of the ground vectors (thus assuring that the end slopes are correct). The remaining rules each attempt to reduce the angles between

adjoining sectors to zero. Together their effect is to create a solution of minimum curvature. 
In order to search for a suitable solution a strategy is adopted to determine a solution that lays between the two possible extremes. These are that a straight blade is maintained (i.e. all joint angles are zero, implying that the endpoint constraint is not met) or the end constraints are perfectly met but with an uncontrolled curvature in between (similar to that of tying a piece of string between the two vectors). This is achieved by firstly weighting the end condition rules to be very much truer than the curvature rules (which were needed to be broken). A ratio of a 100:1 was found by evaluating the desired end accuracy against the likely degree of curvature. During the solution search a further strategy was included. This employs looping through the solution routine with an increasing angular limit to the blade segments (by employing a search variable bounding routine). This effectively commences the search with a 'stiff' blade that is at each cycle made more 'flexible'. In this manner each attempt moves progressively towards the simplest blade curvature (Figure 5). Again it is difficult, if not impossible to find the absolute minimum strain energy, as was the case with the Kallay algorithm. It was found that some shapes are quite fast settling down to the desired end shape, while others keep changing for a much larger number of iterations. Using this algorithm it is therefore also necessary to use proper stopping criteria for the calculations. In this paper this algorithm is referred to as the Medland algorithm

\section{Measuring the shape of a bent flexible blade}

\subsection{The measuring set-up}

A number of experiments was carried out in which a flexible beam was bent to a shape by applying specified constraints on it's extends. The blade was fixed in a certain shape and then measured. For this task a setup was designed, describing the used material for the beam, the manner of fixing the beam and the types of shapes to be

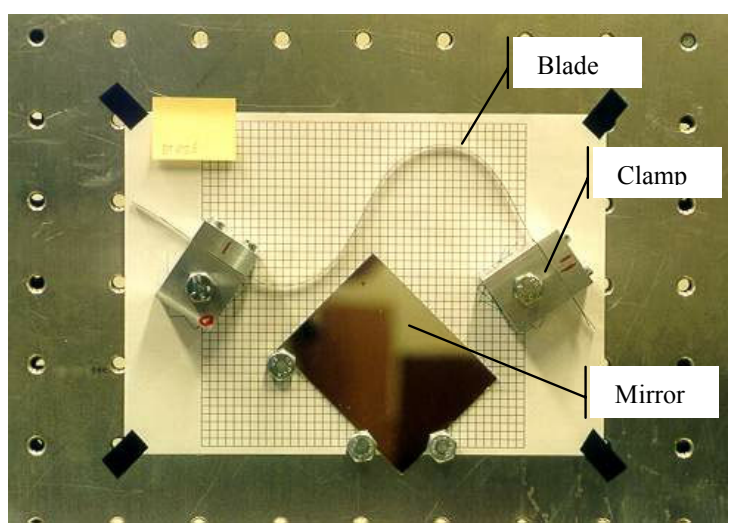

Figure 6. Overview of the measuring set up. measured. An appropriate technique was selected to measure the produced shapes and convert them to a digital format so that they can be compared to calculated shapes. A simple set up was created consisting of an Aluminum breadboard with M10 holes spaced every $50 \mathrm{~mm}$. Two clamping devices are mounted 
that are able to clamp virtually every shape and thickness of material within the spectrum of material dimensions that were used. Figure 6 shows the set-up.

The clamping devices can be fixed in any angular orientation referred to the horizon. The created blade holder has no angle-, length- or distance indication by itself. They have to be measured using external measuring equipment. Using this set-up it is possible to create a series of curved blade shapes that can be measured. Also it is possible to apply heating to the blade by clamping it between electrical insulators and applying a sufficient amount of electrical current through the blade. Again an external measurement is necessary in order to know the actual temperature of the blade.

\subsection{Tested materials}

For the measuring of bending behavior only the elasticity properties of the used material are of importance. If it is necessary to heat the blade a material must be selected that can be heated adequately using electrical power. In the described experiments a dedicated stainless spring steel was applied, defined in Europe with the material number 14310. From this material different cross sections were tested in order to find a blade that combines a reasonable flexibility with enough stiffness to keep its shape when small forces are applied. Strips were tested with cross sections of $5.0 * 0.4,4.0 * 0.3$ and $5.0 * 0.2$ millimeters. The resulting shapes were compared to calculated results, and experiments on the stability of the shape were executed by applying disturbing forces on the blades. It was found that for our set of comparing tests the material 14310 with cross section $4.0 * 0.3 \mathrm{~mm}$. is well applicable. This material was used for the experiments.

\subsection{The investigated set of shapes}

During the experiments a limited set of shapes was measured and calculated. In the

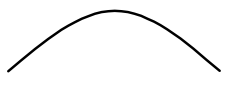
Symmetrical shape

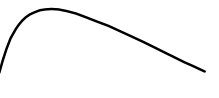

Asymmetrical shape

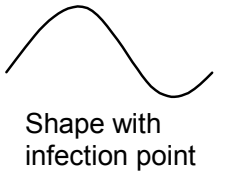

development of the flexible cutting technology

it is planned to use blade shapes with

Figure 7. Shape families that were used in the experiment.

maximum one inflection point because of the needed stability in the blade shape, which cannot be guaranteed in shapes with more than one inflection point. Therefore only U-shaped and S-shaped blades will be allowed. A set of shapes was applied consisting of three more or less symmetrical shapes, three clearly asymmetrical shapes without inflection point, and three shapes containing one inflection point. 
The three variations in each group are 1) slightly curved, 2) moderately curved, and 3) strongly curved.

The setting of the shape is done manually. The operator creates a shape, estimating the amount of symmetry and curvature. Measurements afterwards determine the exact end conditions that were put onto the blade. Figure 7 presents the investigated test set. The difference in the angles $\alpha$ and $\beta$ in the symmetrical shapes is less than 3 degrees, in the asymmetrical shapes more than 10 degrees. The nine selected shapes are measured and then calculated, using the aforementioned algorithms.

\subsection{Measuring method}

The problem of measuring the shape of a thin and flexible beam is not trivial. If a tactile measuring method is used there is a risk of deforming the shape while measuring by the applied probe force. Using a non-tactile method like laser scanning, it is difficult to measure a very thin metal beam that is free standing in space. Laser scanners send out a ray of light and measure from different angles where the reflection of this ray is detected. Laser scanners are known for having problems on scanning reflective surfaces and on detecting edges. Therefore it is not a logical choice to apply a laser scanner for this measuring task. As it is assumed that the blade to take the shape of a planar curve, the needed measuring technique should be capable to perform a matrix measurement in a plane with a size of approximately 250

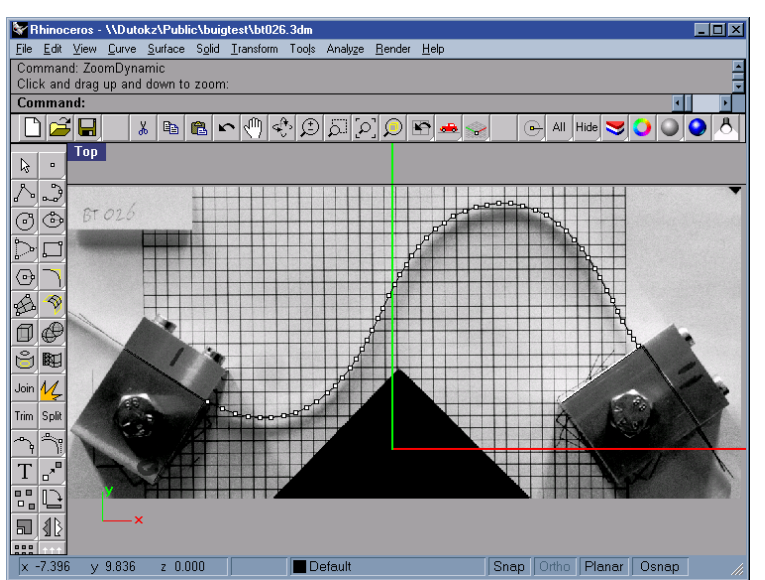

Figure 8. Tracing the scanned image of the blade in a CAD program
* $250 \mathrm{~mm}$. with a resolution of $0.1 \mathrm{~mm}$..

The measuring method that was applied uses photographed images of the blade shape. The photographic image is made while making sure that the viewing direction of the camera is perpendicular to the plane in which the measured curve is situated. This is done by aligning the camera in a mirror that is attached to the set-up in such a way that the center of

the diaphragm is exactly in the center of the viewfinder. The images are digitized, after which the digitized data can be compared to calculated data or to other digitized shapes. A possible problem in this method is the distortion of the optical system of the used camera. This can cause a different image on the film than the actual visible shape. A high quality professional camera with a fixed (opposed to zoom) lens was applied to create the images on film and a digital film scanner to digitize the images. In order to 
determine the accuracy of the film scanner a number of rectangular test shapes was scanned. Evaluation of the resulting pictures showed a maximum error in one direction of $0.09 \%$, which means an error of $0.27 \mathrm{~mm}$ in a working area of 300 times $300 \mathrm{~mm}$. When the horizontal and the vertical direction are

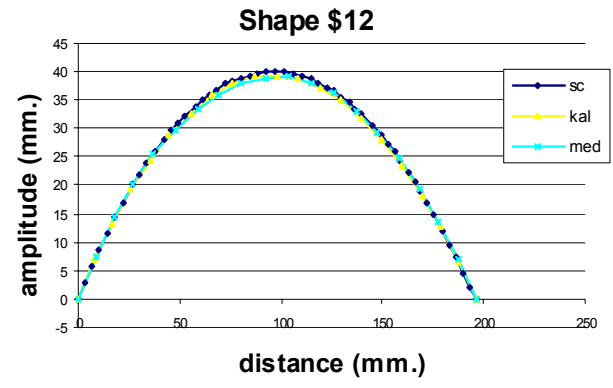

shape $\$ 25$

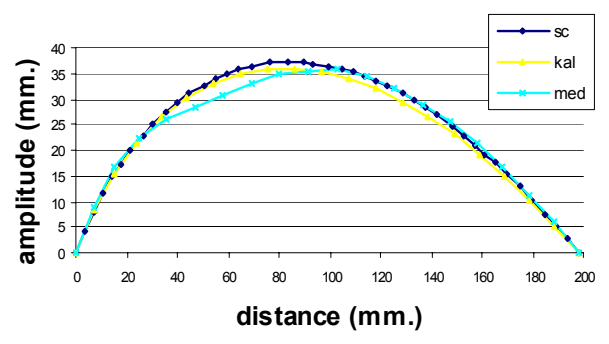

shape $\$ 39$

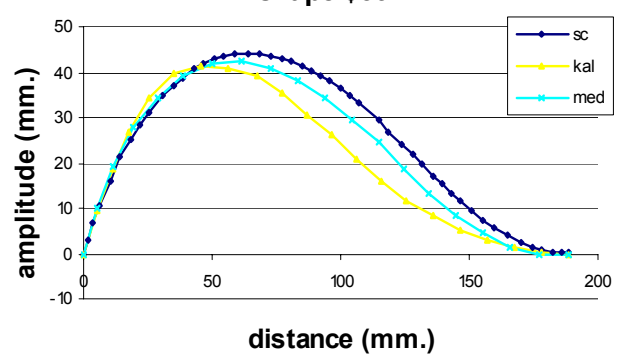

deviations \$12

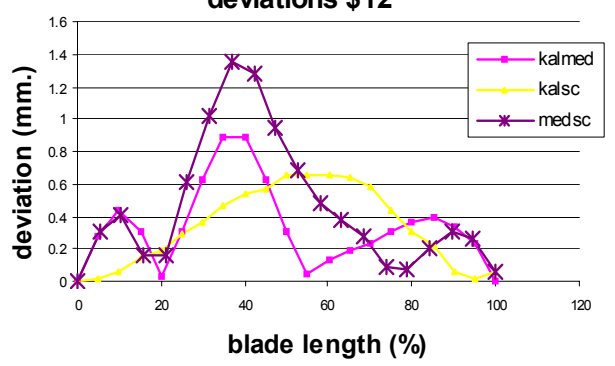

deviations $\$ 25$

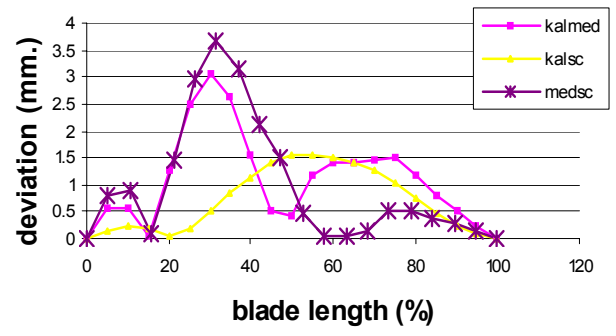

deviations $\$ 39$

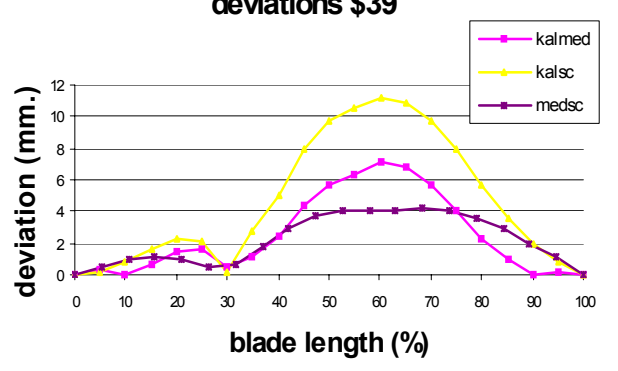

Figure 9. Overview of the measured and calculated averagely curved shapes and their deviations. Blade lengths are all approximately $215 \mathrm{~mm}$.

compared a much larger error of about $0.56 \%$ is found. Therefore a corrective one-dimensional scaling has to be performed on the photographed shapes. After this scaling an accuracy of around $0.3 \mathrm{~mm}$. is reached. Once a blade shape is photographed the edge can be traced manually using a CAD package where the image is used as a background image, thus generating a number of points on the edge (figure 8). After the one-dimensional scaling these points describe the shape of the blade but the size is not yet correct. Therefore a physical measurement must be performed in the original set up that was 
photographed in order to calibrate the scale of the digitized data. When this is done it is possible to compare the measured data with other data sets that were calculated or measured in another way.

\section{Analyzing the calculated and measured results}

The most direct way of comparing calculated and measured results is by plotting the shapes in one graph and interpreting the differences. However when the differences are small this method is not feasible, as the existing deviations are too small in relation to the line thickness of the graphs. Therefore an alternative method was implemented where the differences are better visible. A computer program was developed that is capable to calculate the position of a point on a polyline and the smallest distance from that point to another polyline. Now the deviation between two polylines can be plotted as a function of the length of one of the polylines (Figure 10). A third method to compare the results is by plotting the curvature along the curve. This gives information about the smoothness quality of the produced curves,
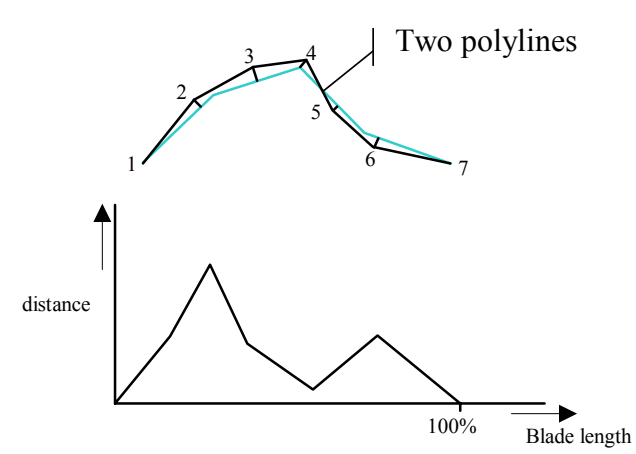

Figure 10. Visualizing deviations as a function of the blade length and also a comparison of energy content can be made. The steps that must be taken to compare physical shapes to calculated data are now 1) create a bitmap image of the physical shape, 2) extract the blade shape from the bitmap by tracing it in a CAD program, 3) export the found polyline and calculate deviations from other polylines, 4) plot the calculated deviations and

curvatures. Figure 9 shows three sets of graphs for averagely curved shapes, namely the symmetrical, the asymmetrical and the inflected shape. From the graphs it becomes clear that the most regular shape $(\$ 12)$ results in the smallest deviations between calculated and measured shapes. Both algorithms, Kallay (kal) and Medland (med), perform reasonably well. The largest deviations are found between the Medland algorithm and the measured values. In the section on tuning the algorithm these specific errors will be dealt with in greater depth. The asymmetric shape (\$25) shows somewhat larger deviations and again the Medland algorithm is the worst performing. In the calculated shape a clear bump is visible where the algorithm apparently stopped optimizing too early. The inflected shape is more difficult for the Kallay algorithm; there the Medland algorithm shows the best result. 
Looking at the curvatures of the measured and calculated shapes in Figure 11 two observations can be made. Firstly it appears that the curvature of the measured shapes (the three graphs on the left) contains quite some noise. This can be understood from the fact that the traced points are quite close together so that a small (human) tracing error results in a large curvature change, which is compensated in the next point. Once the noise is filtered out a clear tendency is visible in the measured curvatures. Secondly it appears that the two calculation algorithms used show considerable differences in local curvatures
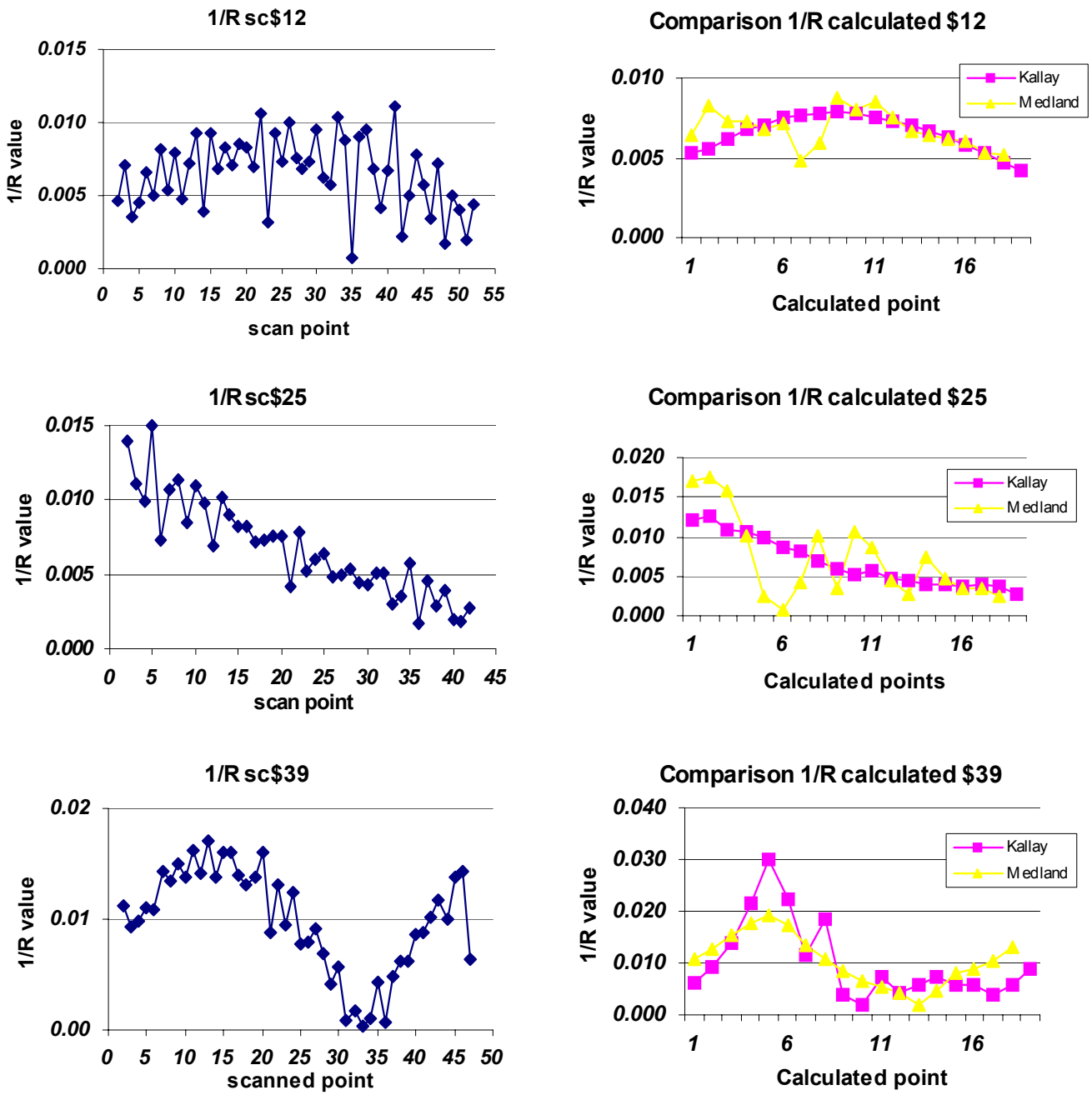

Figure 11. Comparison of curvatures in measured and calculated lines

although the averages again show comparable tendencies. In parallel to the absolute deviation graphs it is found that the Kallay algorithm again performs better on the uninflected shapes, while the Medland algorithm performs best in the inflected case. From these results it can be conclude that the used stop criteria for the calculations are not chosen optimal yet. The Kallay algorithm seems to need more steps to 
properly converge in the inflected case, while the Medland algorithm stopped too early in the other two cases

\section{Meaning of the analysis results for Tool implementation}

In the foregoing paragraphs a method was described to compare calculated blade shapes with measured data. The ability to calculate blade shapes with a known and acceptable accuracy is a first necessity for

sensitivity $\$ 12$

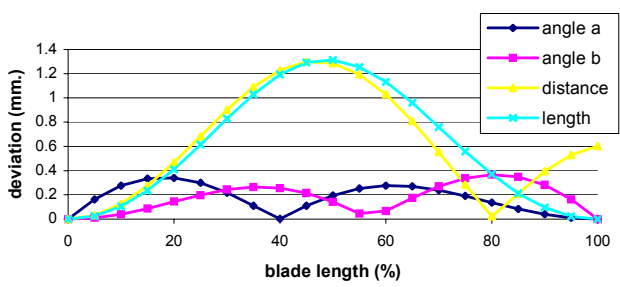

Figure 12. The sensitivity of one shape for a one-unit alteration in each of the input parameters. adequate control of the shape cutting process. A next step is to determine the effect of inaccuracies of the controlling actuators on the total blade accuracy. In a physical tool implementation the input parameters will never be set exactly to the requested values but only within a certain tolerance. There are absolute accuracy issues as well as dynamic synchronization issues that will

cause deviations from the desired settings. In order to be able to predict the impact of different inaccuracies a sensitivity analyses was performed on the three symmetrical shapes. For one of the calculated shapes the four involved parameters were varied with one unit ( $1 \mathrm{~mm}$. or 1 degree), calculated the resulting shapes and compared them to the original. All disturbances are chosen as one unit, that means $1 \mathrm{~mm}$. in length and distance, and one degree in the angles. These disturbances are more or less

relative sensitivityof symetrical shapes

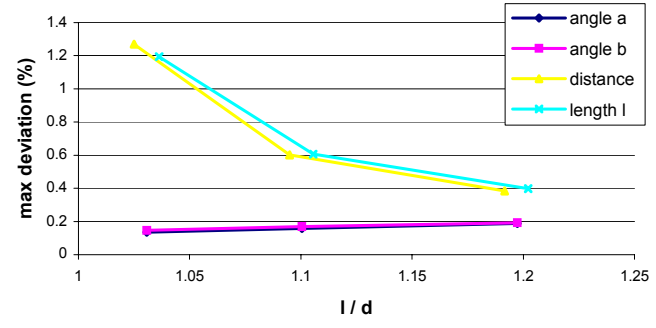

Figure 13. Sensitivity for each of the input parameters in relation to parameters I and $\mathrm{d}$ comparable in terms of actuator quality, which means that a linear actuator with an accuracy of one $\mathrm{mm}$. is comparable in quality to an angular actuator with a one-degree accuracy The plots give a good impression of the effects of disturbances. Figure 12 shows the plot for the averagely curved symmetrical shape $(\$ 12)$.

From the plot it is clear that the two angle

deviations generate comparable but mirrored errors, which was expected. More or less the same is true for the length 1 and the distance $\mathrm{d}$. It is further clear from the graph that the deviations caused by length and distance errors are more prominent than the ones resulting 
from angular errors. This means that the involved linear actuators should be of high quality compared to the angular actuators. In order to determine whether these influences show a linear behavior in the shape domain a plot was created of the maximum deviations as a function of the blade length divided by the connection point distance (Figure 13). The plot shows de deviations as a percentage of the blade length 1 . The graph shows that the deviations caused by angular inaccuracies are slowly increasing with increasing blade length. The deviations caused by length and distance errors start very high and then drop quickly to a level comparable to the initial disturbance (the initial disturbance is $1 \mathrm{~mm}$. and the used blade lengths are all around $200 \mathrm{~mm}$ ). Repeating the analysis with errors of 0.5 units showed that all found deviations halved. This gives ground to the assumption that the sensitivity shows linear behavior in a one-unit margin. That means that this sensitivity analyses data can be applied to define the needed accuracy of our actuators in the tool. Supposed that an accuracy of $0.5 \mathrm{~mm}$. should be reached, firstly the allowed error should be distributed over the available sources. Assuming that in the worst case all errors work in the same direction, the sum of these errors should not exceed $0.5 \mathrm{~mm}$. From the sensitivity analyses graphs it seems reasonable to declare $0.1 \mathrm{~mm}$ to the angle actuators and $0.4 \mathrm{~mm}$. to the linear actuators, so 0.05 $\mathrm{mm}$. per angular actuator and $0.2 \mathrm{~mm}$. per linear actuator. If it is stated state that $1 / \mathrm{d}$ will always be 1.1 or larger, then linear actuators with an accuracy better than $0.33 \mathrm{~mm}$. and angular actuators with an accuracy better than 0.25 degrees should be selected. Note that this is the needed accuracy and not necessarily the needed resolution. 


\section{Tuning of algorithms}

Both applied algorithms use a polyline to approximate the minimal strain energy curve. A difference between the two approaches is that in the Kallay algorithm by definition all the polyline joints behave similarly, while in the case of the Medland algorithm it is possible to define each joint separately by

Shape $\$ 12$ adapted

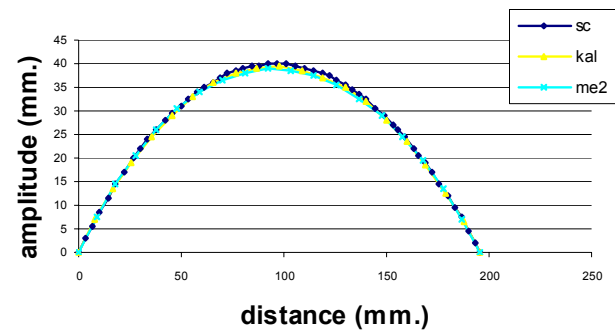

shape $\$ 25$ adapted

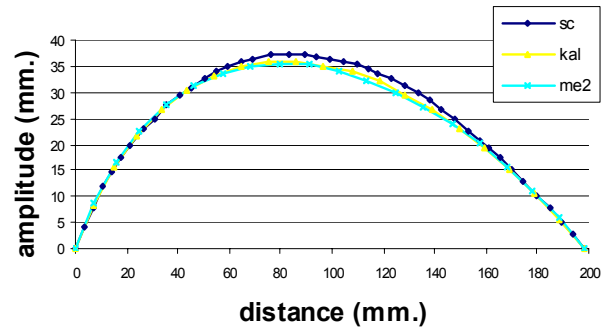

shape $\$ 39$ adapted

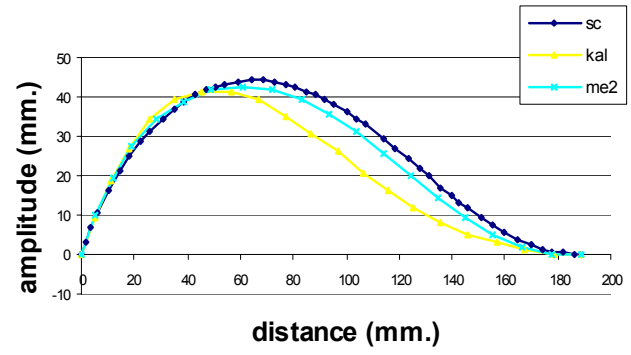

deviations $\$ 12$ adapted

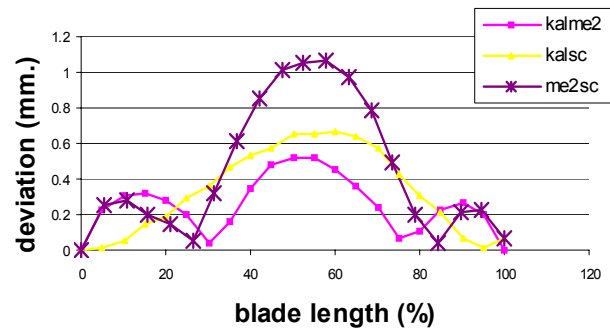

deviations $\$ 25$ adapted

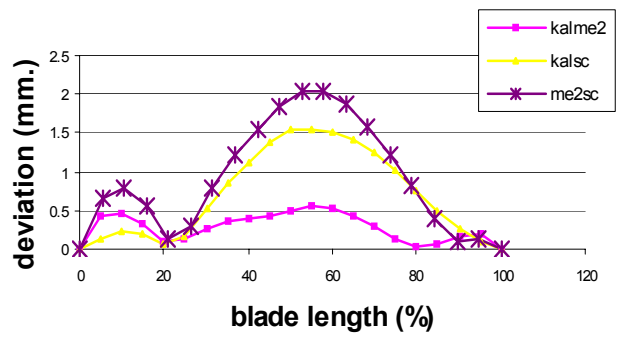

deviations $\$ 39$ adaptet

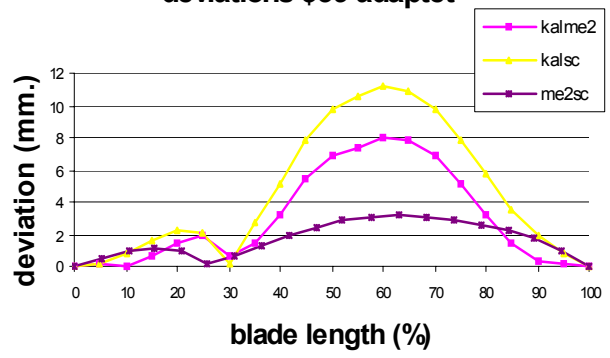

Figure 14. Overview of measured and calculated shapes and their deviations as in figure 9 but with an adapted Medland algorithm

adapting the weight of its curvature rule. This possibility was applied to adapt the Medland algorithm to the measured reality. In the comparison in figure 9 it is visible that the Medland curves show a kind of overshoot in the beginning and the end of the curve. The calculated curve starts too steep and ends too steep compared to the measured one. This can be understood based on the fact that the start and end segment have exactly the direction defined by the input parameters. In reality however the blade starts 
curving immediately and not after one twentieth of the blade. Therefore the weight of the rule that is controlling the begin and end tangents was changed so that it would be more flexible. Further the stop criterion of the algorithm was changed from a fixed number of iterations into a conditional stop that is triggered by the change in truth-value. Once the change in truth-value drops below a given percentage of the former truth-value the process stops. Using this improved implementation the analysis was repeated and the newly found shapes and deviations were plotted(Figure 14). From the graphs it is clear that the Medland results have substantially improved for all shapes but are not optimal yet, The Kallay results have not changed in these plots as the algorithm was not changed. Although only one adaptation of the Medland algorithm was performed we are confident that it can be optimized further.

Secondly two improvements were made in the Kallay implementation namely 1) instead of one iteration process, four processes are started in which the iteration order and direction along the curve are varied. After a limited number of iteration steps the results are compared and the solution with least strain energy is selected. This improvement helps to avoid the local minima in which the iteration can be trapped. The selected part solution is used for further iterations. 2) In our first implementation the amount of possible iterations was limited by the choice for a high conversion speed. This choice was made based on test results with simple shapes. After evaluation of all our results, it was determined that for a more complex inflected shape this limitation is too tight, and causes the iterations to stop in a suboptimal stage. The conversion speed was decreased thus creating the proper conditions for the algorithm to actually stop

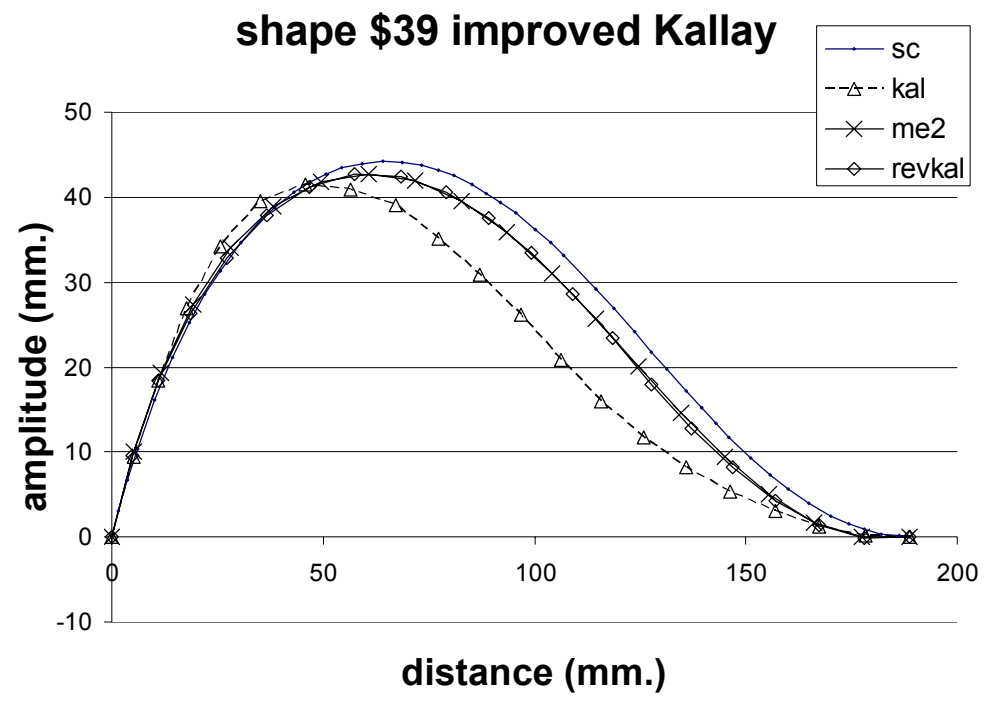

Figure 15. Improved stop criteria for the Kallay algorithm. once no more significant improvements can be made. This improved implementation results in a considerable improvement in the calculated results as we can observe in figure 15 where the revised Kallay algorithm produces virtually the same curve as 
the adapted Medland algorithm.

Apart from the accuracy there are some more criteria that can influence the choice for an algorithm, namely 1) the speed of calculation and 2) the usability in different areas of the shape domain. The calculation times for both algorithms were measured and it was found that the Medland algorithm is substantially slower than Kallay. Because the implementations are not optimized for speed and have run on incomparable machines it is difficult to say how large this difference actually is but for this moment the Kallay algorithm is considered to be the fastest.

The usability over the whole of the shape domain was initially low for both algorithms. The Kallay algorithm worked well in the uninflected area while the Medland algorithm performed best on the inflected shapes. It was found that tuning of the algorithms changed this. Figure 14 shows substantial improvements of the Medland algorithm by only one algorithm change and figure 15 shows a considerable improvement in the Kallay implementation. The examples described above show that by using the developed tools and methods it is possible to quickly evaluate new algorithms or adapted versions and subsequently improve them in an iterative manner.

\section{Conclusions}

We have developed the necessary methods and tools for measuring the shape of a curved thin flexible blade. Further tools were developed for the evaluation and comparison of measured- and calculated shapes. The developed methods and tools were applied in a number of experiments, involving shape measurement, shape calculation and evaluation of the results. It was found that the developed measuring method is well applicable and has in our implementation an accuracy of $0.3 \mathrm{~mm}$. From the results that were found it was concluded that both the algorithms used initially were not directly applicable for our purpose. After implementing adaptations in the algorithms the calculated results were again evaluated and concluded was that the overall quality of both adapted algorithms had been improved significantly. In doing so it was demonstrated that this way of working creates the possibility to evaluate the quality of an implemented shape calculation algorithm and to iteratively optimize it in a verifiable way. Finally in an example it was demonstrated how the analysis tools and methods can be applied for determining the sensitivities for control errors in an implemented tool. In this way they can be used for the final tool specification. 


\section{References}

[1] Bram de Smit, Johan J. Broek, Imre Horváth, Expected performance of the FF-TLOM technology, based on preliminary testing of the involved basic technologies; Proceedings of $1^{\text {st }}$ RAPDASA ANNUAL CONFERENCE, PRETORIA, SOUTH AFRICA, 8 \& 9 November 2000

[2] Thomas, C.L., Gaffney, T.M., Kaza, S. \& Lee, C.H., 1996, "Rapid Prototyping of Large Scale Aerospace Structures", Proceedings of the 1996 IEEE Aerospace Applications Conference, IEEE catalog \#96CH35904, ISBN 0-7803-1396-6.

[3] R.L. Hope, P.A. Jacobs, and R.N. Roth, Rapid Prototyping with Sloping Surfaces, Rapid Prototyping Journal 3-1 (1997) 12-19

[4] de Jager, P.J., 1998, "Development of a New Slicing Methodology to Improve Layered Manufacturing", Ph.D. thesis, Delft University of Technology, pp. 129.

[5] D. G. Ahn, S. H. Lee and D. Y. Yang, “Development of transfer type variable lamination manufacturing (VLM-st) process", International Journal of Machine Tools \& Manufacture, volume 42 (2002), pp. 1577-1587.

[6] Lee, E. H., Forsythe, G. E., (1973), "variational study of nonlinear curves”, SIAM Rev., Vol. 15, No. 1, pp. 120-133.

[7] Y. Takeuchi, K. Morishige and N. Nakakarumai, "Effective 5-Axis Control Machining Using Conicoid End Mill”, In: Rapid Product Development, N. Ikawa, ed., Chapman and Hall, London, (1997), Part 5 Advanced NC, Ch.31 313-322.

[8] I. Horváth, Z. Kovács, J.S.M. Vergeest, J.J. Broek, and A. de Smit, "Free-Form Cutting of Plastic Foams: A New Functionality for Thick-Layered Fabrication of Prototypes", Proceedings of the TCT'98 Conference, Nottingham, (1998) 229-237

[9] Horváth, I., Vergeest, J.S.M., Broek, J.J. \& de Smit, A., "Tool Profile and Path Calculation for Freeform Thick-Layered Fabrication", Computer Aided Design, 30-14 (1999) 1097-1110

[10] I. Horváth, J.J. Broek, Z. Rusák, G. Kuczogi and J.S.M. Vergeest, Morphological Segmentation of Objects for Thick-Layered Manufacturing, Proceedings of the 1999 ASME Design Engineering Technical Conference, paper DETC99/DFM-8932.

[11] Frisch-Fay, R., 1962, Flexible Bars, Butterworth, Washington, D.C. 
[12] Cox, M. G., (1986), "Data Approximation by Splines in One and Two Independent Variables", in NPL Report DITC 7/86, National Physics Laboratory, Teddington.

[13] Horn, B. K. P., (1983), "The curve of least energy", ACM Transactions of Mathematics and Software, Vol. 9., No. 4, pp. 441-460.

[14] Vergeest, J. S. M., (1989), “Surface Fitting for Interactive Shape Design”, Computers in Industry, Vol. 13, No. 1, pp. 1-13.

[15] M. Kallay, Method to approximate the space curve of least energy and prescribed length, Computer Aided Design 19-2 (1987) 73-76.

[16] Medland, A.J., Mullineux, G. and Rentoul, A.H., (1995), "introducing solid objects into a constraint moddeling system", Engineering with computers, 11 (1995), 27-35

[17] Kenny, L.P.J., Rentoul, A.H., Twyman, B.R., Kerr, D.R. and Mullineux, G., (1997) "a software environmet for conceptual mechanism design", proc. Instn. Mech. Engnrs: Part C - J, Mech. Eng. Science, 211 (1997), 617-625 
Figure captions

Figure 1. Example of a multi-layered stacked assembly.

Figure 2. The tool that was build

Figure 3. Shape control parameters

Figure 4. A few steps in the MSE curve generation

Figure 5. Iteration steps during constraint based optimization

Figure 6. Overview of the measuring set up

Figure 7. Shape families that were used in the experiment

Figure 8. Tracing the scanned image of the blade in a CAD program

Figure 9. Overview of the measured and calculated averagely curved shapes and their deviations. Blade lengths are all approximately $215 \mathrm{~mm}$.

Figure 10. Visualizing deviations as a function of the blade length

Figure 11. Comparison of curvatures in measured and calculated lines

Figure 12. The sensitivity of one shape for a one-unit alteration in each of the input parameters.

Figure 13. Sensitivity for each of the input parameters in relation to parameters I and $d$.

Figure 14. Overview of measured and calculated shapes and their deviations as in figure 9 but with an adapted Medland algorithm

Figure 15. Improved stop criteria for the Kallay algorithm 